CALT-TH-2014-142

HDP: $14-03$

\title{
Banjo timbre from string stretching and frequency modulation
}

\author{
David Politzer \\ California Institute of Technology
}

(Dated: June 20, 2014)

The geometry of a floating bridge on a drumhead soundboard produces string stretching that is first order in the amplitude of the bridge motion. This stretching modulates the string tension and consequently modulates string frequencies at acoustic frequencies. Early work in electronic sound synthesis identified such modulation as a source of bell-like and metallic timbre. And increasing string stretching by adjusting banjo string-tailpiece-head geometry enhances characteristic banjo tone. Hence, this mechanism is likely a significant source of the ring, ping, clang, and plunk common to the family of instruments that share floating-bridge/drumhead construction.

$* * * * * * * * * * * * * * * * * * * * * * * * * *$

PACS: 43.75.Gh

key words: banjo, frequency modulation, floating bridge, tailpiece

contact info: politzer@theory.caltech.edu, (626) 395-4252, FAX: (626) 568-8473; 452-48 Caltech, Pasadena CA 91125

separate figures: FIG1-politzer.eps, FIG2-politzer.eps 


\section{What is a Banjo?}

A banjo is a drum with strings mounted on a neck. With minor caveats, that is what makes it a banjo. So that is what must be responsible for its characteristic sound. Actu-

ally, the banjo is the American instrument fitting that description. ${ }^{1,2,3}$ Cultures around the world have their own versions. While there is great variation among their voices, they are acoustically identifiable as belonging to the banjo family. Among the many are the akonting and kora of west Africa, the sarod of India and its neighbors, the dramyin of Tibet, the dashpuluur of Tuva, and the shamisen of Japan.

A reasonable question is: what is it in the mechanics of sound production by drum and strings that distinguishes banjo sound from that of other stringed instruments? While it may not be easy to quantify the defining characteristics of that sound, "Ring the banjo" is a phrase used and commonly understood in America since before the Civil War, an era when banjos had no metal parts.

\section{Geometry of Break Angle and String Stretch}

A possible answer lies in the geometry of how the strings are attached, how they go over the bridge, and how the bridge moves.

The simple, ideal, textbook string with fixed ends must stretch as it vibrates. However, the amount of stretch is second order in the amplitude of vibration. The textbook analysis typically ignores this stretching and arrives at a description of normal modes and frequencies that gives a very satisfactory account for most musical situations. Of course, it is possible to pluck a guitar string with such ferocity that the initial sound is, in fact, distorted by the stretching.

The floating bridge on a drumhead produces a different behavior with respect to stretch. "Floating" refers to the bridge's relation to the strings. Specifically, the floating bridge goes up and down relative to the ends of the strings, which are fixed to the rim and the neck. That is to be contrasted, for example, with a bridge and saddle, as on a flat-top guitar, where the bridge end of the string goes up and down with the bridge.

"Break angle" is the angle the strings make going over the bridge. It is determined by the bridge height and tailpiece geometry, as roughly illustrated in FIG. 1. (Here and in what follows, American banjo terminology is used to describe the various parts and motions. However, all instruments in this world-wide family have analogous parts, e.g., some way to 
do the same job as the tailpiece to anchor the string ends to the edge of the drum.)

String tension is determined by scale length (nut to bridge), string gauge (or density), and chosen pitch of the open string. To a good approximation, the tension is uniform from the tuners to where the string is anchored on the tailpiece. The string tension produces a downward force on the bridge. When the bridge is at rest, this is canceled by the upward force of the distorted head.

In FIG. 2, $L$ is the scale length (bridge to nut), $l$ is the bridge to tailpiece distance, and $\theta_{o}$ is the equilibrium break angle. The equilibrium length of the string is

$$
S_{o}=L+l / \cos \theta_{o} .
$$

If the bridge moves up a distance $x$, the string must stretch a length

$$
\Delta S=\sqrt{L^{2}+x^{2}}-L+\sqrt{l^{2}+\left(l \tan \theta_{o}+x\right)^{2}}-\sqrt{l^{2}+\left(l \tan \theta_{o}\right)^{2}} .
$$

In practice $x$ is much smaller than $l$. For example, $x$ could be $0.1 \mathrm{~mm}$ and $l$ could be $4 \mathrm{~cm}$. Using $x \ll l$ :

$$
\Delta S \simeq x \sin \theta_{o}
$$

As $\theta_{o} \rightarrow 0$ (and $x \ll l$ ) there remains a stretch proportional to $x^{2}$, i.e., yet smaller by a factor of $x / l$. (The stretch that accompanies the normal transverse vibration along the string itself is likewise proportional to the square of the amplitude of that vibration.)

\section{From Stretch to Frequency Modulation}

Localized stretch and changes in tension propagate along a string at the longitudinal speed of sound in the material. For steel strings, that speed is roughly 20 times greater than the speed of transverse waves in normally tuned strings. Hence, it is reasonable to approximate the stretch as producing an instantaneous increase in tension. If a given stretch were applied once and for all, there would be a corresponding rise in pitch. If the stretching happened very slowly, one could still think of the stretch as a change in pitch, i.e., an adiabatic change.

Strings of different materials have different stretching moduli. In particular, steel strings are much stiffer (longitudinally) than gut, nylon, or other synthetics. Since it is the drumhead that moves air, the sound volume is a function of the magnitude of the bridge motion. So, for a given sound volume, steel strings experience greater changes in tension than nonmetallic strings.

Strings of different gauges mounted on a particular banjo will experience different changes in tension for a given bridge motion. However, the fractional pitch changes will be about 
the same for all strings of the same material because the tuned pitches are proportional to the square root of the ratio of tension to density.

If tension changes while a string is vibrating, it is an inherently non-linear system. Some care is then required when thinking in terms of Fourier components. In particular, it is the entire bridge motion that modulates a given string's tension, and, typically, that bridge motion is not sinusoidal but, rather, has many strong Fourier components.

The important picture to take from this discussion is that each string's tension is modulated by the motion of the bridge, and that motion is roughly periodic with the period of the lowest notes being played. This manifests acoustically because each string's frequencies, harmonics, and partials are proportional to the square root of its modulated tension.

\section{The Sound of Frequency Modulation}

In 1973, Chowning published his discovery of the sound of frequency modulation. ${ }^{4}$ In music, sub-audio frequency modulation gives a familiar form of tremolo. (Common usage obscures a traditional distinction between tremolo and vibrato.) On stringed instruments, for example, the player can stretch the string periodically, a few to several times per second, and the pitch will go up and down. (This is accomplished by pushing the fretted or fingered string sideways or pushing on a whammy bar.) Using a music synthesizer, Chowning found that, when the frequency of the modulation is increased and itself enters the audio range, the warble disappears, and it is the timbre of the note that is effected. The originally dull, sinusoidal, signal-generator sound becomes brighter, more metallic, and bell-like when subjected to audio range frequency modulation. The abstract mathematics is the same as for FM radio signals. ${ }^{5}$ Only here, the relevant frequencies are not several orders of magnitude apart. The modulation induces frequency sidebands along with the original signal, spaced on the order of the modulation frequency. From ref. [5], "As the index sweeps upward, energy is swept gradually outward into higher order side bands; this is the originally exciting, now extremely annoying 'FM sweep'. The important thing to get from these Bessel functions is that the higher the index, the more dispersed the spectral energy - normally a brighter sound." Bessel functions give the explicit frequency spectrum of sine functions modulated by sine functions. And "index" is the relative frequency range of the modulation.

It is worth noting that frequency modulation gives the smoothest possible signal consistent with a given frequency spectrum. Frequency spectrum does not uniquely determine 
the sound, and human hearing is sensitive to time-domain features on remarkably short scales. The bright sound under discussion is specifically the one that results from frequency modulation.

\section{Observational Support}

Three kinds of observational support are readily available for this perspective on banjo sound. The first requires access to modern math software such as Mathematica ${ }^{\circledR}$. You can construct your own functions of time and then listen to them. In particular, you can easily listen to the sound of sinusoidal modulations of sinusoidal functions and even add an amplitude envelope typical of plucked string sound. ${ }^{6}$ Of course, it will not sound like a banjo. A huge number of details are missing. But the extra ring and brightness of tone stands out.

A second demonstration requires a computer sound program, such as the freely distributed, open-source Audacity ${ }^{\circledR}$, which allows you to record and speed up the recording. Play a low note on a banjo and push down periodically (perhaps 6 to 12 times per second) on the head near a foot of the bridge. That will produce an audible frequency tremolo. Speed up the recording until the modulation frequency is well above $20 \mathrm{~Hz}$. (36 Hz should do.) The sound will have acquired a definite metallic plink, akin to banging on sheet metal. This is most dramatic if the original note was quite low and the original break angle as small as possible. (Adjustment of tailpiece or choice of tailpiece can accomplish the latter.) Actually, this demonstration can be performed with any low note frequency tremolo from any instrument - except that you would miss the connection to bridge motion.

The third category of support comes from very well-established, universally agreed upon lore among banjo players. Without any agreement on why or how, experienced banjo players and builders know that break angle is an important issue. Some tailpieces are adjustable over a range with the turn of a screw, while others produce a fixed break angle, whose value depends on the banjo on which it is mounted. The range on currently popular instruments is roughly $6^{\circ}$ to $15^{\circ}$.

Often, the tailpiece advice comes with the observation that a steeper angle produces a greater down-pressure of the strings on the bridge. However, at equilibrium, that force is canceled by an upward force of the head. Furthermore, the string-head system acting on the bridge supplies the same return force as a function of bridge displacement as with a shallower 
angle - at least over the relevant range of angles and assuming the head force on the bridge is linear with displacement over the range of bridge motion. (It is essential to remember that the strings are retuned to their original tensions after the tailpiece is adjusted.)

So, even if the mechanism is not widely understood, the consequence is: increasing the break angle makes the sound more banjo-like. The words that are often used to describe the sound of higher angles are: "sharper," "snappier," or "brighter," while lesser break angles produce "mellow," "warm," or "round tone." 7 It is not that gut strings with gentle break angles are not banjo-like. It is just that steel strings with sharp break angles are more so. In fact, some bluegrass pickers shy away from the most extreme setting of the tailpiece, stopping short of the aspect of frequency modulation that Schottstaedt ${ }^{5}$ termed "extremely annoying."

\section{Contrast with Other Stringed Instruments}

There are other acoustic, stringed instruments with floating bridges, where the bridge moves relative to the fixed ends of the string. These include the violin family, mandolins, and arch-top guitars. However, their bridges, riding on wooden soundboards, do not move nearly as much as the bridge on a banjo for the same sort of pluck. For example, the violin, with a soundboard that has around $94 \%$ the area of a typical banjo, produces a far quieter sound when plucked. Also, the quintessential banjo features disappear if we replace the skin on a banjo with wood. Such instruments exist. They may be called banjos if they are are strung and played like banjos, but their sound is far quieter, sustain is far longer, and tone rather dulcimer-like.

\section{Non-linearities from the Drumhead Itself}

While drumheads were likely initially chosen for soundboards because of the sound volume produced, they also make a characteristic sound. Some of that comes from their interaction with string tension via the bridge. But there may well be other non-linearities inherent in

the use of a drumhead that contribute to that sound, as well. This deserves further study, but some basic issues are clear.

The dynamics of the head are relevant both for how the whole head vibrates in response to driving by the bridge and how the head pushes back on the bridge. The non-trivial stress tensor of a banjo head, even at equilibrium, is apparent to the player, particularly in the vicinity of the bridge. The head is certainly not a fluid membrane, whose only restoring force 
comes from a uniform, isotropic tension. In addition, it is also possible that typical motions of the head in the vicinity of the bridge go beyond the range in which the relation of stress to strain can be linearized. This is an additional possible mechanism for the brightening of sound by the generic drum/string system.

\section{Conclusion}

Any particular instrument in the banjo family has features responsible for its characteristic timbre. And banjo players, in particular, are known for their penchant for adjusting and swapping parts in a quest for some ideal sound. However, all these instruments have a pluck which identifies them as banjo-like and distinguishes them from anything else. First-order string stretching and the consequent frequency modulation contribute to that distinctive sound.

\section{Acknowledgement}

I would like to thank Frank Rice of Caltech for constructive observations.

\section{References}

1. J. Dickey, The structural dynamics of the American five-string banjo, J. Acoust. Soc. Am., 114 (5), 2958 (2003)

2. J. Rae, Banjo, in The Science of String Instruments, T. Rossing, ed., ch. 5 (Springer, New York, 2010)

3. L. Stephey \& T. Moore, Experimental investigation of an American five-string banjo, J. Acoust. Soc. Am. 124 (5), 3276 (2008)

4. J. Chowning, The Synthesis of Complex Audio Spectra by Means of Frequency Modulation, J. Audio Eng. Soc. 21 (7), 526 (1973)

5. B. Schottstaedt, An Introduction to FM, https://ccrma.stanford.edu/software/snd/snd/fm.html

6. J.-C. Risset and M. Matthews, Analysis of Musical Instrument Tones, Physics Today $22(2), 23(1969)$

7. R. Siminoff, How to Set Up the Best Sounding Banjo, (Hal Leonard, Milwaukee, 1999).

\section{Figure Captions}

FIG 1: schematic of break angle, tailpiece, string, bridge, and head

FIG 2: break angle $\theta_{o}$ and bridge motion $x$ determine stretch 


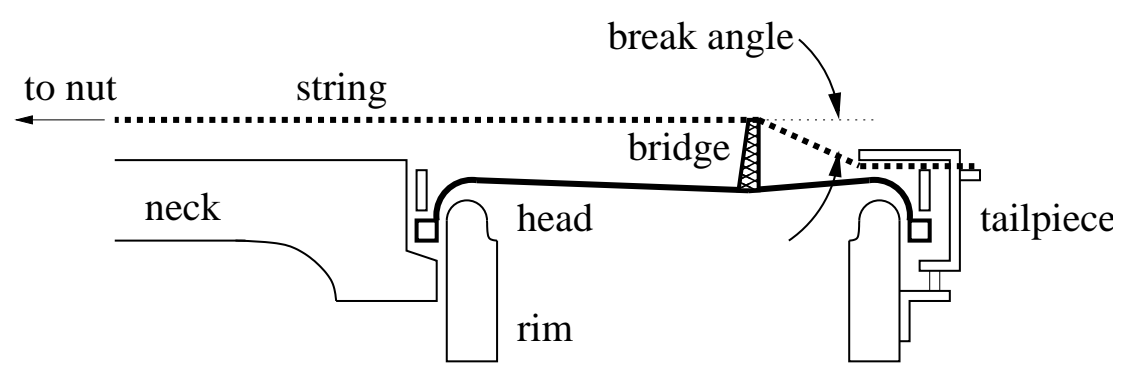

FIG. 1. schematic of break angle, tailpiece, string, bridge, and head

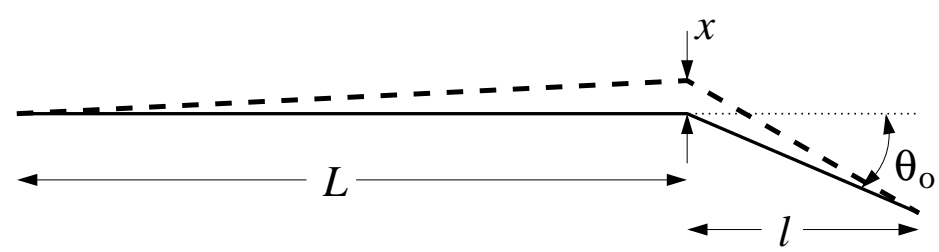

FIG. 2. break angle $\theta_{o}$ and bridge motion $x$ determine stretch 